\title{
V-ITS: Video-based Intelligent Transportation System for Monitoring Vehicle Illegal Activities
}

\author{
Vehicle Intelligent Transportation System by Abbas Q
}

\author{
Qaisar Abbas \\ College of Computer and Information Sciences, \\ Al Imam Mohammad Ibn Saud Islamic University (IMSIU), Riyadh 11432, Saudi Arabia
}

\begin{abstract}
Vehicle monitoring is a challenging task for videobased intelligent transportation system (V-ITS). Nowadays, the V-ITS system has a significant socioeconomic impact on the development of smart cities and always demand to monitor different traffic parameters. It noticed that traffic accidents are exceeded throughout the world with the percentage of $1.7 \%$. The increase in accidents and the percentage of deaths are due to the people that don't abide by the traffic rules. To address these challenges, an improved V-ITS system is developed in this paper to detect and track vehicles and driver's activities during highway driving. This improved V-ITS system is capable to do automatic traffic management that saves traffic accidents. It provides the feature of a real-time detection algorithm for driver immediate line overrun, speed limit overrun and yellow-line driving. To develop this V-ITS system, a pre-trained convolutional neural network (CNN) model with 4-layer architecture was developed and then deep-belief network (DBN) model was utilized to recognize illegal activities. To implement $\mathrm{V}$ ITS system, OpenCV and python tools are mainly utilized. The GRAM-RTM online free data sets were used to test the performance of V-ITS system. The overall significance of this intelligent V-ITS system is comparable to other state-of-the-art systems. The real-time experimental results indicate that the VITS system can be used to reduce the number of accidents and ensure the safety of passengers as well as pedestrians.
\end{abstract}

Keywords-Computer vision; intelligent traffic management system; traffic monitoring; vehicle tracking from video; image processing; deep learning

\section{INTRODUCTION}

An intelligent transportation system based on the video (VITS) automatically tracks the vehicle illegal driving activities is an active research area in the field of computer vision and socioeconomic development. Due to rapidly increase the vehicles, the intelligent system is required to control serious injuries caused by traffic-related accidents. This problem is widespread across the globe. In fact, the automatic tracking of a vehicle is required to monitor the roads and highways as well. To monitor the road or highways, there are many traffic parameters that should be calculated such as over-speeding, yellow-lane or off-road driving and detection of obstacles presented on the road. Such an expert system also facilitates driver assistance during automatic driving.

In particular, the statistics of accidents in Saudi Arabia is increasing rapidly during the last five months. In one estimation, there were 82.281 accidents in one month. These accidents were due to not use of leaving enough safety distance between vehicles, run red traffic lights, sudden change of lane, and lack of commitment to the priorities. The system is predicted and discovered the mistakes of the drivers especially highway driving. A desktop application software was developed to detect and track both vehicle and driver's activities during highway driving, using a real-time video camera installed on highway road.

This V-ITS based system communicates with the respective authorities to apply strict measures when the driver exceeds the speed limit or track sudden change or leave out the yellow lane or stop in the left path. On the road, the activities tracking is a challenging and important task due to influence in surveillance or road site accidents. In the past studies, the surveillance cameras [1] on the road are increasing due to the negligence of the car or truck drivers during highway driving. Therefore, there is a great demand to increase surveillance systems.

The main goal of this paper is to propose the latest computational intelligence algorithms using deep-learning concept and to develop an effective and efficient system for providing vehicle illegal activities detection rate from video sequences. The use of video image processing and machine learning algorithms for traffic monitoring was initiated in many countries. When these algorithms have implemented on the hardware, there are different parameters calculated. All video detection systems used for traffic monitoring can be broadly classified in two categories: 1) Systems which rely on localized incident detections, and 2) Systems which track individual vehicles. The advantage of the first is that the computational requirements are quite low, and algorithms are relatively simple. In the case of vehicle tracking systems, sophisticated algorithms are needed and are usually computationally demanding. As a result of this project, our main objective is to track vehicle activities during highway driving time. Vehicle tracking systems offer a more accurate estimation of microscopic traffic parameters like lane changes, erratic motion etc.

In V-ITS system, detection and monitor is planning to develop to track activities of vehicles on the spot by using realtime video sequences. Generate a penalty if there is a traffic violation occur during the driving process on the highways. Automatically discover the most times and days accidents, and peak traffic violations. Predict accidents and congestion during highway driving. Automatic generation of Penalties to illegal drivers. If use ITS-V system then the drivers will try to control 
the speed limits that will definitely reduce accidents on the road. Compare to the speed limit, the accidents are occurred to immediate line turn or driving on yellow-line to cross the vehicles very fast. In case of different weather conditions on the road, the chances of accidents will reduce to $70 \%$ due to the immediate line overrun, speed limit overrun and yellow-line driving.

\section{RELATED WORK}

Object tracking from live video sequences in Video surveillance [1] applications is an important and emerging research area, which is attracting many scientists. The object tracking is directly related to the domain of computer vision under image processing category. It has many applications in practice such as traffic control, security, and surveillance and mass events, etc. To effectively track an object, it is always a challenging task. During driving, some ways have to find out to track the vehicle activities so that the accidents should be minimized. Also, object tracking is required to highlights the role of humans in the next generation [2] of driver assistance and intelligent vehicles. It is important to detect and track the activities of human or robotic driving for safety reasons.

It noticed that the statistics of road-side accidents are rapidly increasing throughout the world especially in Saudi Arabia. According to estimation, there were 82.281 accidents occurred during a time period of one month. These accidents were due to not use of leaving enough safety distance between vehicles, run a red traffic light, sudden change of lane, and lack of commitment to the priorities. In this paper, an automatic system is developed to predict and discover the mistakes of the drivers, especially highway driving. A desktop application software is implemented to detect and track both vehicle and driver's activities during highway driving, using a real-time video camera installed on highway road. Real-time car detection and tracking are applied over hundreds of image frames. The proposed system will communicate with the respective authorities to apply strict measures when the driver exceeds the speed limit or track sudden change or leave out the yellow lane or stop in the left path.

In fact, the road, traffic lights and other drivers on the road [3] information are provided by the vision-based systems. It noticed that the number of vehicles increased nowadays and created a total burden on computer vision systems. Therefore, there is a dire need for developing effective and efficient solutions for tracking vehicle illegal activities on the road. Moreover, the security risks have significantly increased and that becomes an important subject for law enforcement authorities for surveillance highways. To solve these problems, there are lots of researchers who develop a tracking algorithm but still inefficient and un-effective.

Another requirement of these systems in public is to have a rationally large number of pixels on an objective. There are many different types of objectives which could be important and it is often not possible to get a large number of pixels on objective and robustness [4]. The road digital cameras must provide plenty related to uncommon events compared to just over speed information. For example, those digital cameras must include plenty of information related to immediate line overrun, speed limit overrun and yellow-line driving.
Automatic detection and identification of objects is the main importance of security systems and video surveillance applications. Automatic video surveillance is placed to provide coverage over the scenes of most interest. Within the scope of view of the camera, some areas are of greater importance than others and some areas are really of no interest at all.

For an automatic development of vehicle detection and tracking, it is an important subject in the domain of computer vision. Therefore in this paper, a system is trying to develop an application that can track vehicle activities from live video streaming during highway driving. The primary aim of this project (ITS-V) is to focus on the detection of moving vehicles for surveillance purposes. This problem is also related to the domain of artificial intelligence [5] due to recognizing moving objects from live video streams. When working on ITS-V problem, an accurate solution is required to track object and segment [6] it at the same time without losing time efficiency. Also, real-time vehicle activity tracking means that the tracking and segmentation step were integrated together. It is also important that the developed application must help to law enforcement agencies in case of discovering any vehicle violating laws and it helps in improving traffic safety levels and raise the quality of existing roads network.

The features of the system can achieve a better standard of safety on the roads to reduce the rate of accidents and deaths among drivers as less as possible. It can read the exceed speed limit of the vehicle. The system will work on highways only because according to statistics accidents often happen on the highways. To reduce the number of accidents, a system is developed that works in good control with the vehicle, so it can follow the driver's movements and behaviors. And the system processes the images of accidents in the video clips and adds them to a dataset to discover which roads have the most accidents.

The speed limit parameter for a traffic violation in KSA is varied from $120 \mathrm{~km}$ to $140 \mathrm{~km}$ per hour if the driver goes over it more than $10 \%$ the system will detect him. In the past studies, the authors utilized some image processing and machine learning algorithms to detect real-time driver's activities. Those machine learning techniques such as SVM [7], [8], PCA [9], Neural Networks [10] or Bayesian decisionmaking [11]. However, in this paper, the latest deep learning techniques are integrated together to develop this novel system.

Instead of driving activities control system, there is also a requirement for developing driving assistance systems and that is considered to be a challenging task $[12,13]$. To do this task, there are many algorithms proposed to track the vehicles in the motorway driving [14]. The advantage of these systems is their real-time performance. There are many systems also talking about the problems related to detect and track real-time vehicle activities. These systems are explained below.

An intelligent transportation system based on the video (ITS-V) is providing an important solution for the problem of socioeconomic [15] impact on society. For tracking or segmentation of vehicles, there is a need to monitor different traffic parameters such as yellow-line, off-road or immediate line changing during highway driving. Since, there are lots of challenges of traditional video-based tracking systems such as 
in case of vehicle drifting, occlusions, obstacles and detection still in various environmental conditions. To address these challenges, there are few studies that focused on all these problems during vehicle tracking.

Another paper [16], the authors developed a driving assistance system to track head-and eye-blinking. Whereas in [17], the authors presented a solution to detect a lane using a vision system on the vehicle. Though, they concluded that lane detection is a difficult problem because of the varying road conditions that one can encounter while driving. There are also some papers that talking about the techniques for monitoring and understanding of real-world human activities, in particular of drivers, from distributed vision sensors [18]. In order to achieve this goal, the authors used different parameters such as head pose estimation module, hand, and foot tracking, egovehicle parameters, lane, and road geometry analysis, and surround vehicle trajectories. The system is evaluated on a challenging dataset of naturalistic driving in real-world settings.

In contrast to these approaches, the authors developed an Intelligent Vehicle Monitoring System [19] that using Global Positioning System along with Google Maps and Cloud Computing which collects useful information about a vehicle. The detection of a vehicle is also a challenging task. In paper [20], the authors developed a video-based analysis system that detects, tracks and archives vehicles in video stream data at multiple resolutions. This step is important even for controlling activities of autonomous vehicle driving. Even in urban areas [21], the sensors utilized in the car to communicate with the clouds to given information about drivers activities during driving. In this study [22], the authors introduced an activity classification system based on activity class through random forests (RFs) classifier. Moreover, in [23], the authors discussed a human-centered perspective to develop an intelligent vehicle.

There are a lot of limitations and deficiencies of the current strengths and weaknesses of existing V-ITS approaches of video-based vehicle tracking, which are as under. Previous approaches did not provide effective traffic monitoring results due to the effects of occlusion and spillover. The state-of-theart approaches utilized old fashion image processing and machine learning algorithms to track the vehicle illegal movements. In addition, large trucks often occlude neighboring vehicles and make them hard to recognize side-by vehicle activity. Though the approach is this paper to overcome these limitations. The past studies have the inability to detect vehicles due to headlight reflections and different weather situations such as dust storm. The dust storm is come in KSA and making the automatic system totally raise false positive to detect vehicle activities.

The accuracy of tracking is also affected by the distance of the camera from the closest lane. A larger pan angle is required to cover all lanes when the camera is placed far from the closest lane, so the camera should be placed as close to the closest lane as possible. By using previous techniques, the selection of key points of vehicles is crucial and those systems are sensitive to drift and occlusion. The other developed systems outside the KSA for automatically determining the vehicle illegal activities are computationally expensive due to the increasing number of vehicles. It noticed from the literature that there is a dire need to develop a high-performance vehicle detection method.

To overcome these above-mentioned problems, an improved V-ITS system was presented through advanced deep learning algorithms to get robust results. In the past studies, the deep learning algorithms [24-36] have many variants to represent visual features such as the convolutional neural network (CNN), recurrent neural network (RNN), deep belief networks (DBN), restricted Boltzmann machine (RBM) and AutoEncoder. A four multilayer convolutional neural network (CNN) [38] model is trained in different roads samples along with diverse environmental conditions. Those samples were obtained from GRAM Road-Traffic Monitoring (GRAMRTM) dataset [37]. The three layers in the CNN model were dedicated towards driver immediate line overrun, speed limit overrun and yellow-line driving along with one features extraction layer. After this CNN model, a deep belief neural network (DBN) [39] model was used to classify these three drivers' illegal activities.

\section{METHODOLOGY}

The systematic flow diagram of all steps of the proposed VITS system is displayed in Fig. 1. An increasing number of vehicles and environment conditions created a new interest in the development of new technologies in real-time video image processing. It noticed from the past studies that there are many commercial systems proposed but they have difficulties with congestion, shadows, dust storms, and lighting transitions. Also, there are some illegal driving activities such as yellowlane driving that they are unable to detect. Therefore in this research study, a feature-based transform system is developed to overcome these difficulties by using advanced deep-learning algorithms to process real-time video sequences. The author of this paper entitled this system as a video-based intelligent transportation system (V-ITS).

In this paper, the vehicle features are tracked instead of tracking just entire vehicles to make it fit for various environmental conditions such as occlusion and lighting conditions. Also, the developed system can easily differentiate between cars and trucks through feature-based tracking. Moreover, there are some examples of training samples for traffic violations on a highway are shown in Fig. 2.

Overall the proposed vehicle tracking system is developed based on four main stages that are mentioned below. To develop this ITS-V system, the firsts step is to segment of the scene into individual vehicles and then tracking each vehicle inside a tracking zone. A simple background subtraction technique was utilized to segment vehicle from the background video frames. After detecting and segmenting the vehicle form a background scene, the next step is to compute traffic parameters such as vehicle speed in different lanes of the highway roads. After collecting local parameters thorough CNN model at the collection site, this intelligent data is then passed on to automated and operator assisted applications, which is developed through the DBN model for classification. In the end, plenty is generated according to the specific traffic 
violation and stored in the somewhere distributed server to avoid further traffic violations.

The first step of this research study is to extract the video frames from the side of highway daily time driving. In this paper, a pre-train four multi-layer convolutional neural network (CNN) model was used to transform features for prediction of driver's illegal activities during highway driving. Those frames are extracted from a video in every 0.3 seconds. Afterward, a deep-belief network (DBN) model was applied to predict the final decision of the activity class of driver's illegal driving. The DBN model is trying to help pre-train CNN model to pool layer for defining an effective features map. In the proposed system, a single features map was utilized that was extracted from a single video frame. To perform the final prediction about the driver's illegal activities, a group of frames was used for recognition. From the video frames, fifteen feature maps are stored to generate by the pre-trained $\mathrm{CNN}$ model for prediction, the equivalent of three seconds of video. Afterward, this group of feature maps is concentrated into one single pattern, which will be the input of our proposed DBN model, to obtain the final classification of the traffic violation system.

To train the CNN model, the GRAM road-traffic monitoring (GRAM-RTM) dataset was utilized. In this dataset, the sample videos are selected according to different environmental conditions to make the system runs better compare to state-of-the-art systems. The methodological steps are explained in the upcoming subsection of this paper.

\section{A. Acquisition of Datasets}

GRAM Road-Traffic Monitoring (GRAM-RTM) dataset [37] was utilized in this paper to test and compare the performance of proposed V-ITS system. In fact, the GRAMRTM dataset consists of multiple vehicles tracking ground truth during real-time video processing. The V-ITS system was tested and implemented on HP brand Laptop with an Intel core processor of the processing capability of i7 CPU @ $3.35 \mathrm{GHz}$ and $8 \mathrm{~GB}$ of RAM with Windows XP. This program was programmed in OpenCV and Python tools. The experimental results were also statistical measured. The dataset of 1200 region-of-interest (ROI) video frame images including normal of 600 and traffic violation of 600 were acquired from GRAMRTM to test and evaluate the performance of V-ITS system. An example of this dataset from urban second video is visually displayed in Fig. 2.

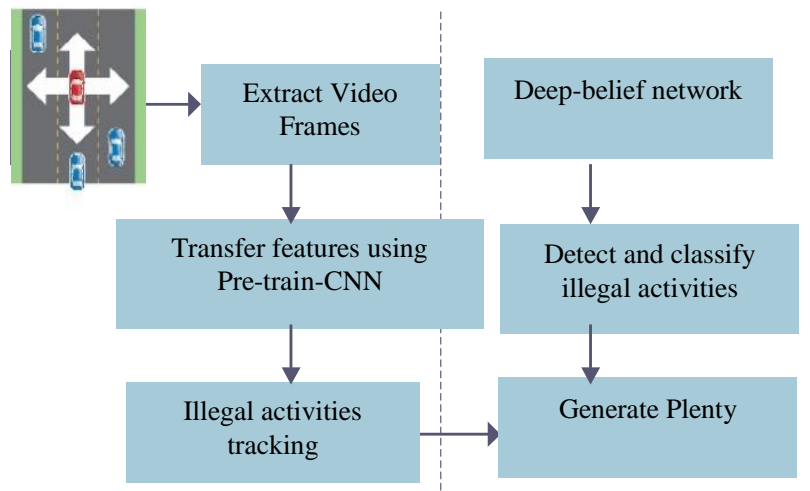

Fig. 1. A Systematic Diagram of Proposed V-ITS System for Detecting Vehicle Illegal Activities.

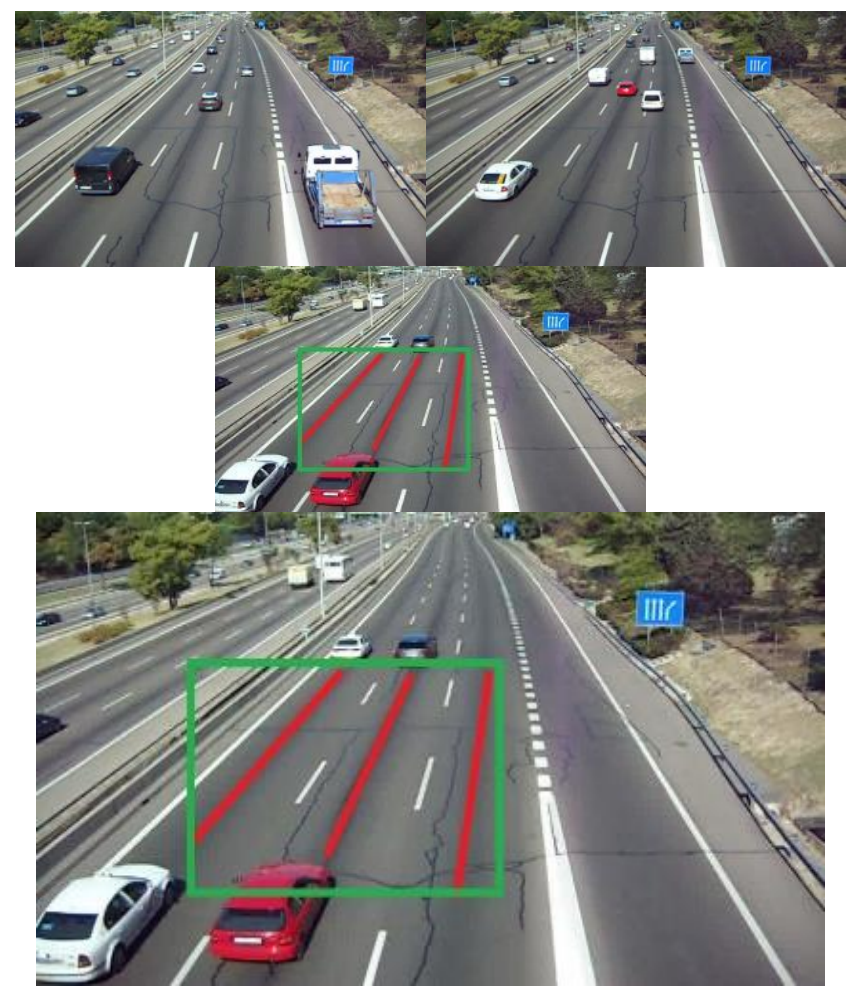

Fig. 2. An Example of Training a Convolutional Neural Network Model for Transfer Training Video Region-of-Interest (ROI).

The region-of-interest (ROIs) for each video frames from GRAM-RTM dataset is automatically defined to trained the convolutional neural network (CNN) [38] for transform features. In the video frames, the three-lines are drawn to defined vehicle tracking and checking illegal activities. This step is visually represented in Fig. 2. The high-level features are defined from these ROI video frames to effectively train the multilayers of the CNN model. The detailed description of this $\mathrm{CNN}$ model is defined in the next subsection.

\section{B. Pretrain CNN Model}

Convolutional neural network (CNN) model [38] is using in many applications to extract deep features and it has applications in many different fields. In practice, the CNN model is used to detect the pixel-level features using convolutional filters in different layers and then finally classify them through softmax linear classifier. In the past studies, the authors achieved significantly higher performance than manually tune machine learning algorithms.

To extract features from an image, a domain-expert knowledge is required to detect best features from image processing. However, if a convolutional neural network (CNN) model is applied to a digital image then it provides deepinvariant features. Those features are extracted from a different multilayer of CNN model. To recognize the objects from images, the features-map is responsible for the output layer. But the features-map created by CNN model is not optimized for real-time video processing. Therefore, a pre-train CNN model was utilized to get an effective features map.

In this paper, a pre-train CNN model is utilized to extract effective and optimize features from video frames. A pre- 
training strategy was used to guide $\mathrm{CNN}$ for defining the features from video frames. The dataset is divided into three different groups such as immediate line overrun, speed limit overrun and yellow-line driving. The frames are extracted from GRAM Road-Traffic Monitoring (GRAM-RTM) dataset that defined the problems of immediate line overrun, speed limit overrun and yellow-line driving. In fact, the $\mathrm{CNN}$ network structure is learned to recognize the three different tasks for a traffic violation from labeled video sequences. At the top layer of the CNN model, the features are extracted and transformed into weights to train the next layer of the CNN model. A pretraining step for $\mathrm{CNN}$ multilayer architecture along with deepbelief network (DBN) is explained in the subsequent paragraphs.

The region-of-interest (ROIs) are extracted from video frame from fixed regions and then send to the four multilayer $\mathrm{CNN}$ architecture which has been already trained for high-level feature extraction in different frames from GRAM-RTM dataset video frames in three different categories. Those categories are related to a traffic violation. Convolution and max-pooling layers are added to the network for extracting and selecting the most important features. The two fully-connected deep belief network (DBN) layers are then added to the network after convolution and the pooling layers. In practice, a multi-layer RBM network is utilized for developing in a DBN unsupervised network. In deep learning algorithms, the DBN architecture proved to be an excellent generative model that can easily outperform for fine-tune parameters.

During the training stage, high-level features are learned simultaneously with the training of the proposed CNN network. After four layers of convolution, RBM, and pooling, the features are out of the last layer into the two-layer fullyconnected network for further training. Finally, the trained feature matrix is taken as the input of DBN. DBN is trained and fully connected to the output of the network to predict saliency. In the inference stage, the full image was used as the input of the network. Similar to the training stage, the highlevel features of the test video frame is extracted via the trained CNN network. Finally, using the trained DBN and the learned features, the plenty value was computed in each frame.

In order to develop a pre-train CNN model, the high-level features are learned or extracted together to train the multilayer architecture of the network. In advance step, the pretraining step is applied to get and learn good informative features extracted from video frames. The dataset samples are divided into three category classes. The ROI regions are defined earlier from each video to do better initialization of the training step. These ROIs frames are visually represented in Fig. 2. In three different traffic violation, a set of informative features are defined to effectively train the CNN model. Then a single features map was generated from every single image, which is convolved with a Gaussian mask.

The first layer of convolution neural network (CNN) model was generated by following the DBN and max-pooling concepts. To develop this pre-train $\mathrm{CNN}$ model, the features are obtained at the first layer are additionally learned through the next three CNN network layers. Training Similar to the pretraining step, the training of the proposed CNN network is performed with features from the pre-training step as the input on the same collected from target frame regions.

\section{Driver's Illegal Activities Prediction}

Given a frame region, the driver's illegal activity is predicted through the pre-train CNN model and classifies those activities by deep-belief network (DBN) multilayer architecture. In order to achieve the high-level traffic violation parameters, the ROI image region was used from the video frame to do perfect training of the $\mathrm{CNN}$ network. In fact, the convolutional filters are performed to each layer of the pretrain CNN network model. Afterward, the deep belief network (DBN) model was applied followed by the pooling layer to classify driver's illegal activities. In order to recognize driver's illegal activities, the last two layers are fully-connected layer and input to this layer is a feature map to the DBN architecture. After repeated running a DBN model, a weighted matrix is obtained that is conforming to the high-level video features of this frame. Hence, the traffic violation value of this frame can be obtained via multiplying the weight with the features and it is defined by the Eq. (1).

Plenty $y_{i=1,2,3}^{n}=\max _{f}\left(W_{L} x ; 0\right)$

Where w parameter is learned parameters from the deepbelief network (DBN) classifier for three categories of plenty class, and $\mathrm{x}$ is the high-level features matrix extracted by the well-trained a multilayer convolutional neural network (CNN) network model. In this equation, $G$ parameter denotes the Gaussian masking template to detect driver immediate line overrun, speed limit overrun and yellow-line driving.

\section{EXPERIMENTAL RESULTS}

Experimental results are described in this section to validate the performance of the proposed V-ITS system for detecting vehicle illegal activities without performing pre- or post- image processing techniques. The proposed V-ITS system based best variants of deep-learning multilayer architecture is proposed in this paper that is different from state-of-the-art detection systems.

The performance was evaluated based on the frames that are extracted from GRAM Road-Traffic Monitoring (GRAMRTM) dataset. The multi-layer $\mathrm{CNN}$ architecture model was trained in three different samples from GRAM-RTM dataset. The three different samples driver's illegal activities are counted based on immediate line overrun, speed limit overrun and yellow-line driving. The samples datasets are considered to have different environments such as normal, sunny, and cloudy. To extract high-level features from video frames, the RBM layer was added to pre-train CNN model. To show the effectiveness of proposed pre-train CNN and DBN models, the comparisons with the CNN network is presented in Table I.

The V-ITS system was implemented in python and OpenCV tools in Windows XP 64-bit system. The learning rate for training the four-layer $\mathrm{CNN}$ is initialized as $(10 \times 6)$ with a batch size of 32. The training of the four-layer of CNN model for about 75 epochs and the training procedure costs nearly 12 hours in all. In the experiments, it averagely takes 0.254 seconds to train an image, and 0.152 seconds to test a video frame image. The performance detector calculates two kinds of 
errors, namely to miss a true violation detection and to detect the false violation. The first error is measured by the detection rate while the second one is called the number of false accepts. These measures are represented in an average form in Table I.

To evaluate the performance of V-ITS system, different vehicles are considered in the experiments. The experimental results are shown in Table I. In this table, all types of environmental conditions are considered. As shown in this table, the proposed system is performed better results compared to a simple convolutional neural network (CNN) model with pre-training through principal component analysis (PCA) technique. On average, the proposed V-ITS system is getting $90 \%$ better detection rate compared to the $84 \%$ value obtained by CNN model. The V-ITS system is capable of detection and recognition the traffic violation up to four frames per second (fps), so at normal speed (i.e., $100 \mathrm{~km} / \mathrm{h}$ ), the system offers at least two opportunities to identify driver's illegal activities. In fact, the V-ITS will be upgraded in the future to add more traffic violation at lower speeds. The V-ITS system has been tested in a real-time environment mode during nighttime and daytime under different environmental conditions.

TABLE I. RESULTS CONCERNING THE AVERAGE PRECISION OF TRACKING METHOdS FOR VeHICLES IN SELECTED DATASET FROM GRAMRTM IN CASE OF IMMEDIATE LINE OVERRUN, SPEED LIMIT OVERRUN AND YELLOW-LINE DRIVING

\begin{tabular}{|l|l|l|l|}
\hline \multirow{2}{*}{ No. } & \multicolumn{2}{|l|}{ V-ITS Performance } \\
\cline { 2 - 4 } & Methods & CNN & Pre-train CNN \& DBN \\
\hline 1 & Line-overrun & 0.751 & 0.890 \\
\hline 2 & Limit-overrun & 0.845 & 0.920 \\
\hline 3 & Yellow-drive & 0.821 & 0.945 \\
\hline \multicolumn{2}{|c|}{ Average Detection Rate } & $\mathbf{8 4 . 5 0 \%}$ & $\mathbf{9 0 . 0 1 \%}$ \\
\hline
\end{tabular}

In this paper, a novel V-ITS system based on a new CNN framework is proposed to automatically detect driver's illegal activities during highway driving. To detect effective features, high-level features are learned and the classification results are predicted through the DBN model. The proposed pre-train model is distinct from the state-of-the-art techniques, an extra layer was added through DBN into the $\mathrm{CNN}$ framework to obtain more accurate features. Moreover, to avoid manual annotation of video frames data, Deep Belief Network (DBN) classifier was added to pre-train $\mathrm{CNN}$ model for recognizing of driver's illegal activities without using complex methods of image processing algorithms. The proposed V-ITS system outperforms compare to simple pre-train CNN model using PCA in the same selected dataset.

\section{CONCLUSIONS}

Tracking of vehicle illegal activities is a critical step for the development of an automatic traffic management system. In the past studies, there are many authors focus on extracting traffic parameters without focusing on environmental conditions. In this paper, an efficient V-ITS system is developed to predict the driver's illegal activities during highway driving. The system was evaluated and tested on GRAM-RTM dataset. The experimental results indicate that the proposed V-ITS system outperformed compared to state-ofthe-art video processing systems. It is happened due to use of pre-train $\mathrm{CNN}$ model to transform the features and then DBN is deployed to classify the vehicle illegal activities in multiple video frames. In this paper, the V-ITS system measured traffic parameters due to pre-train $\mathrm{CNN}$ deep learning algorithm to get robust results without any problem or delay. The main objective of this paper is to consider the latest deep learning algorithm to calculate traffic illegal parameters without focusing pre- or post-processing steps as done in many studies. In this study, the combination of transform features and multilayer architecture of deep learning algorithms (DBN) are effectively integrated for better classification results. To implement and test this V-ITS system, Python, computer vision OpenCV tools were utilized. In a future study, more traffic violation is added according to the type of vehicle.

\section{ACKNOWLEDGMENT}

The author would like to thank Deanship of Scientific Research at Al Imam Mohammad ibn Saud Islamic university, Saudi Arabia, for financing this project under the grant no. (380902).

\section{REFERENCES}

[1] S. Ojha and S. Sakhare, "Image processing techniques for object tracking in video surveillance- A survey," International Conference on Pervasive Computing (ICPC), Pune, pp. 1-6, 2015.

[2] E. Ohn-Bar and M. M. Trivedi, "Looking at Humans in the Age of SelfDriving and Highly Automated Vehicles," in IEEE Transactions on Intelligent Vehicles, vol. 1, no. 1, pp. 90-104, March 2016.

[3] J. Wu and X. Zhang, "A PCA Classifier and its Application in Vehicle Detection," in Neural Networks, Proceedings. IJCNN '01. International Joint Conference on, vol. 1, 2001.

[4] S. Sivaraman and M. Trivedi, "A General Active-Learning Framework for On-road Vehicle Recognition and Tracking," IEEE Trans. on ITS, vol. 11, no. 2, june 2010.

[5] A. Broggi, P. Cerri, and P. Antonello, "Multi-resolution Vehicle Detection using Artificial Vision," in Intelligent Vehicles Symposium, 2004 IEEE, june 2004.

[6] H. Grabner, M. Grabner, and H. Bischof, "Real-Time Tracking via Online Boosting," in Proc. BMVC, pp. 6.1-6.10, 2006.

[7] C. Papageorgiou and T. Poggio, "A Trainable System for Object Detection,” Int. J. Comp. Vision, vol. 38, no. 1, pp. 15-33, Jun. 2000.

[8] Z. Sun, G. Bebis, and R. Miller, "On-road Vehicle Detection using Gabor Filters and Support Vector Machines," in 14th Intern. Conf. on Digital Signal Processing (DSP), vol. 2, pp. 1019-1022, 2002.

[9] J. Wu and X. Zhang, "A PCA Classifier and its Application in Vehicle Detection," in Neural Networks, Proceedings. IJCNN '01. International Joint Conference on, vol. 1, pp. 600-604 vol.1, 2001.

[10] N. Matthews, P. An, D. Charnley, and C. Harris, "Vehicle Detection and Recognition in Grayscale Imagery," Control Engineering Practice, vol. 4, no. 4, pp. 473-479, 1996.

[11] H. Schneiderman and T. Kanade, "A Statistical Method for 3D Object Detection applied to Faces and Cars," in IEEE Conf. on Computer Vision and Pattern Recognition, vol. 1, pp. 746-751, 2000.

[12] A. Ess, K. Schindler, B. Leibe, and L. Van Gool, "Object Detection and Tracking for Autonomous Navigation in Dynamic Environments," The International Journal of Robotics Research, vol. 29, 2010.

[13] E. Richter, R. Schubert, and G. Wanielik, "Radar and Vision-based Data Fusion - Advanced Filtering Techniques for a Multi-object Vehicle Tracking System," in Intelligent Vehicles Symposium, 2008 IEEE, pp. $120-125$, , june 2008. 
[14] NC Mithun, T Howlader, SMM Rahman, Video-based tracking of vehicles using multiple time-spatial images, Expert Systems with Applications, vol. 62, no. 15, pp. 17-31, 2016.

[15] C. Braunagel, E. Kasneci, W. Stolzmann and W. Rosenstiel, "DriverActivity Recognition in the Context of Conditionally Autonomous Driving," 2015 IEEE 18th International Conference on Intelligent Transportation Systems, Las Palmas, pp. 1652-1657, 2015.

[16] A. A. Assidiq, O. O. Khalifa, M. R. Islam and S. Khan, "Real time lane detection for autonomous vehicles," 2008 International Conference on Computer and Communication Engineering, Kuala Lumpur, pp. 82-88, 2008.

[17] Eshed Ohn-Bar, Ashish Tawari, Sujitha Martin, Mohan M. Trivedi, On surveillance for safety critical events: In-vehicle video networks for predictive driver assistance systems, Computer Vision and Image Understanding, vol. 134, pp. 130-140, May 2015.

[18] Dimil Jose, Sanath Prasad, V.G. Sridhar, "Intelligent Vehicle Monitoring Using Global Positioning System and Cloud Computing", Procedia Computer Science, vol. 50, pp. 440-446, 2015.

[19] W Wu, EA Bernal, RP Loce, ME Hoover, "Multi-resolution video analysis and key feature preserving video reduction strategy for (realtime) vehicle tracking and speed enforcement systems", S Patent 8,953,044, 2015.

[20] M. Gerla, E. K. Lee, G. Pau and U. Lee, "Internet of vehicles: From intelligent grid to autonomous cars and vehicular clouds," IEEE World Forum on Internet of Things (WF-IoT), Seoul, pp. 241-246, 2014.

[21] Lijie Xu and Kikuo Fujimura, "Real-Time Driver Activity Recognition with Random Forests. In Proceedings of the 6th International Conference on Automotive User Interfaces and Interactive Vehicular Applications (AutomotiveUI '14). ACM, New York, NY, USA, Article 9, 8 pages, 2014.

[22] E. Ohn-Bar and M. M. Trivedi, "Looking at Humans in the Age of SelfDriving and Highly Automated Vehicles," in IEEE Transactions on Intelligent Vehicles, vol. 1, no. 1, pp. 90-104, March 2016.

[23] Jianwei Ding, Yongzhen Huang, Wei Liu, and Kaiqi Huang, "Severely Blurred Object Tracking by Learning Deep Image Representations, IEEE TRANSACTIONS ON CIRCUITS AND SYSTEMS FOR VIDEO TECHNOLOGY", vol. 26, no. 2, FEBRUARY 2016.

[24] Hong yang Xue, Yao Liu, Deng Cai, Xiaofei He, Tracking people in RGBD videos using deep learning and motion clues, Neurocomputing vol. 204, pp. 70-76, 2016.

[25] Bohan Zhuang, Li jun Wang, Huchuan Lu, "Visual tracking via shallow and deep collaborative model”, Neurocomputing, vol. 218, pp. 61-71, 2016.

[26] Naiyan Wang, Dit-Yan Yeung, "Learning a deep compact image representation for visual tracking", Proceeding NIPS'13 Proceedings of the 26th International Conference on Neural Information Processing Systems, Lake Tahoe, Nevada, pp. 809-817, 2013.
[27] Lijun Wang, Wanli Ouyang, Xiaogang Wang, and Huchuan Lu, "Visual Tracking with fully Convolutional Networks", EEE ICCV 2015.

[28] Chao Ma, Jia-Bin Huang, Xiao kang Yang, Ming-Hsuan Yang, "Hierarchical Convolutional Features for Visual Tracking", Proceeding ICCV '15 Proceedings of the 2015 IEEE International Conference on Computer Vision (ICCV), pp. 3074-3082 December 07 - 13, 2015.

[29] H. Li, Y. Li and F. Porikli, "DeepTrack: Learning Discriminative Feature Representations Online for Robust Visual Tracking," in IEEE Transactions on Image Processing, vol. 25, no. 4, pp. 1834-1848, April 2016.

[30] Charissa Ann Ronao, Sung-Bae Cho, "Human activity recognition with smartphone sensors using deep learning neural networks", Expert Systems With Applications, vol. 59, pp. 235-244, 2016.

[31] Trumble, M., Gilbert, A., Hilton, A., Collomosse, "J.: Learning markerless human pose estimation from multiple viewpoint video", In: Proc. ECCV Workshops, 2016.

[32] Lishen Pei, Mao Ye, Xuezhuan Zhao, Tao Xiang Tao Li, Learning spatio-temporal features for action recognition from the side of the video, Signal, Image and Video Processing January, vol. 10, no. 1, pp 199-206, 2016.

[33] Konstantinos Charalampous, Antonios Gasteratos, "On-line deep learning method for action recognition", Pattern Analysis \& Applications, vol. 19, no. 2, pp. 337-354, May 2016.

[34] Cheng-Sheng Chan, Shou-Zhong Chen, Pei-Xuan Xie, Chiung-Chih Chang, Min Sun, "Recognition from Hand Cameras: A Revisit with Deep Learning", Computer Vision - ECCV 2016 Volume 9908 of the series Lecture Notes in Computer Science pp. 505-521, September 2016.

[35] Yanming Guo, Yu Liu, Ard Oerlemans, Songyang Lao, Song Wu, Michael S. Lew, "Deep learning for visual understanding: A review, Neurocomputing", Recent Developments on Deep Big Vision, vol. 187, pp. 27-48, 26 April 2016.

[36] Guerrero-Gómez-Olmedo, R., López-Sastre, R. J., Maldonado-Bascón, S., \& Fernández-Caballero, A. "Vehicle tracking by simultaneous detection and viewpoint estimation", In International Work-Conference on the Interplay Between Natural and Artificial Springer, Berlin, Heidelberg, pp. 306-316, 2013.

[37] G.W. Yang, and J. Hui-Fang, "Multiple Convolutional Neural Network for Feature Extraction," International Conference on Intelligent Computing, pp. 104-114. Springer International Publishing, 2015.

[38] A. Qaisar, "DeepCAD: A Computer-Aided Diagnosis System for Mammographic Masses Using Deep Invariant Features," Computers, vol.5,pp.1-15,2016.

[39] Qaisar Abbas, "Glaucoma-Deep: Detection of Glaucoma Eye Disease on Retinal Fundus Images using Deep Learning", International Journal of Advanced Computer Science and Applications, 8(6):, pp. 41-45,2017. 